\title{
A 6th-order Alternating Group Iterative Scheme for Two Point Boundary Value Problem
}

\author{
Yumin Shi* and Peiying Zhao \\ School of Mathematic and Quantitative Economics, Shandong University of Finance \&Economics Shandong China \\ ${ }^{*}$ Corresponding author
}

\begin{abstract}
In this paper, a new sixth order alternating group iterative (AGI) scheme is derived based on the two point boundary value problem. The scheme is capable of parallel computation; sixth order accurate in space .The numerical examples show that this AGI scheme are more accurate than the fourth order alternating group iterative scheme. It shows that the analysis is correct and the algorithm is feasible and efficient.
\end{abstract}

Keywords—sixth order alternating group iterative (AGI) scheme; two point boundary value problem; finite difference method ; convergence

\section{INTRODUCTION}

The two point boundary value problem is one of the most classic problems in the numerical approximation of differential equations. The goal of this paper is to study appropriate sixth order difference scheme suitable for parallel computation about the two point boundary value problem. Two major types of schemes which are capable of parallel computation are: the alternating schemes ([1]-[6]), and the domain decomposition schemes ([7]-[8]).Our interest is on the alternating scheme which the sixth order NAGI scheme belong.

Before getting into the detail construction of the NAGI scheme, we like to briefly mention some existing alternating scheme. They are: the Alternating Group Explicit (AGE) strategy ([1]), which is proposed by D. J. Evans and A. R. B. Abdullah; and the Alternating Segment Explicit-Implicit (ASEI) scheme ([3]). In these schemes are capable of parallel computation; however, its truncation error is only second order or lower. In [9], a fourth order Alternating Group Iterative (AGI) algorithm was constructed for two point boundary value problem. Thus we propose to derive a new alternating group iterative method, which has truncation error of sixth order.

he sixth order AGI scheme is derived based on the following boundary value problem with periodic solution:

$$
L u=-\frac{d^{2} u}{d x^{2}}+q u=f, \quad a<x<b
$$

$$
u(a)=u(\mathrm{a}+H)
$$

Here $q, f$ are given real continuous functions on $a \leq x \leq b$ and $H=b-a$ represents the length of one period, with $q \geq 0$.

The outline of the paper is as following. The sixth order AGI scheme is derived and its truncation error is obtained, all in Section II. Its convergence is proved in Section III. The numerical examples are presented in Section IV. Finally, a short conclusion remark is given in Section V.

\section{The SIXTH ORDER AGI SCHEME}

Throughout the rest of the paper, $h=H / J$ is used to represent the special size and $J$ is a positive integer: $u_{i}, q_{i}, f_{i}$ denote the finite difference approximate value of $u\left(x_{i}\right), q\left(x_{i}\right), f\left(x_{i}\right)$ respectively. The finite difference scheme is constructed as follows:

$$
\begin{aligned}
& -\frac{2 r}{180} u_{i+3}+\frac{27 r}{180} u_{i+2}-\frac{270 r}{180} u_{i+1}+ \\
& \left(\frac{490 r}{180}+q_{i}\right) u_{i}-\frac{270 r}{180} u_{i-1}+\frac{27 r}{180} u_{i-2}-\frac{2 r}{180} u_{i-3}=f_{i} \\
& \text { Where } \quad r=\frac{1}{h^{2}}, i=1,2, \cdots, J .
\end{aligned}
$$

The truncation error at point $x=x_{i}$ for (3) is:

$$
R_{i}=-\frac{h^{6}}{560}\left[\frac{d^{8} u}{d x^{8}}\right]_{i}+o\left(h^{7}\right) .
$$

We can rewrite the linear difference (3) in matrix form

$$
A u=F
$$

where $u=\left(u_{1}, u_{2}, \cdots, u_{J}\right)^{T}, F=\left(f_{1}, f_{2}, \cdots, f_{J}\right)^{T}$.

We assume that $J=6 k$, and splitting the matrix $A$ into the sum of three matrices 


$$
A=G_{1}+G_{2}+\sum
$$

$$
\text { Where } \quad G_{1}=\frac{r}{360} \operatorname{diag}\left(Q_{6}^{(1)}, Q_{6}^{(2)}, \cdots, Q_{6}^{(k)}\right)
$$$$
\Sigma=\operatorname{diag}\left(q_{1}, q_{2}, \cdots, q_{J}\right)
$$

and

$$
G_{2}=\frac{r}{360}\left[\begin{array}{cccccccc}
Q_{3}^{(1)} & & & & & -4 & 54 & 540 \\
& Q_{6}^{(2)} & & & \ldots & & -4 & 54 \\
& & Q_{6}^{(3)} & & & & -4 \\
& & & & & & & \\
& & & \ddots & & & \\
-4 & & & & \ddots & & \\
54 & -4 & & & & & Q_{6}^{(k)} & \\
-540 & 54 & -4 & \ldots & & & Q_{3}^{(s)}
\end{array}\right] \text {. }
$$

Here ,

$$
\begin{gathered}
Q_{6}^{(i)}=\left[\begin{array}{cccccc}
247 & -270 & 27 & -4 & & \\
-270 & 490 & -270 & 54 & -4 & \\
27 & -270 & 733 & -540 & 54 & -4 \\
-4 & 54 & -540 & 733 & -270 & 27 \\
& -4 & 54 & -270 & 490 & -270 \\
& & -4 & 27 & -270 & 247
\end{array}\right], i=1,2, \cdots, k .^{\prime} \\
Q_{3}^{(1)}=\left(\begin{array}{ccc}
733 & -270 & 27 \\
-270 & 490 & -270 \\
27 & -270 & 247
\end{array}\right) Q_{3}^{(s)}=\left(\begin{array}{cccc}
247 & -270 & 27 \\
-270 & 490 & -270 \\
27 & -270 & 733
\end{array}\right) .
\end{gathered}
$$

By using (4) and (5), we have

$$
\left(G_{1}+G_{2}+\Sigma\right) u=F
$$

Thus the sixth order AGI scheme can be expressed into the following vector form:

$$
\left\{\begin{array}{l}
\left(\rho I+\bar{G}_{1}\right) \tilde{u}^{(k+1)}=\left[\left(\rho I-\bar{G}_{2}\right) u^{(k)}+F\right] \\
\left(\rho I+\bar{G}_{2}\right) u^{(k+1)}=\left[\left(\rho I-\bar{G}_{1}\right) \tilde{u}^{(k+1)}+F\right]
\end{array} k=0,1,2, \cdots\right.
$$

where $\bar{G}_{1}=G_{1}+\frac{1}{2} \sum, \bar{G}_{2}=G_{2}+\frac{1}{2} \sum, \quad \rho$ is a positive iteration parameter and $\left(\rho I+\bar{G}_{1}\right)^{-1},\left(\rho I+\bar{G}_{2}\right)^{-1}$ exist.
III. THE CONVERGENCE ANALYSIS

Lemma 1 If $A=\left(a_{i j}\right) \in C^{m \times n}$, then

$\|A\|_{2}=\sqrt{\lambda_{1}}, \quad \lambda_{1}$ is the max eigenvalue of $A^{H} A$.

According to lemma 1 , if $A$ is real and symmetric matrix, then $\|A\|_{2}=\rho(A), \rho(A)$ is the spectral radius of $A$.

In (7) $\bar{G}_{1}$ and $\bar{G}_{2}$ are both positive definite matrices. The convergence of (7) can be obtained next.

Eliminating $\tilde{u}^{(k+1)}$ of (7), we have

$$
u^{(k+1)}=T u^{(k)}+\tilde{F}
$$

here

$$
\begin{aligned}
& T=\left(\rho I+\bar{G}_{2}\right)^{-1}\left(\rho I-\bar{G}_{1}\right)\left(\rho I+\bar{G}_{1}\right)^{-1}\left(\rho I-\bar{G}_{2}\right) \\
& \tilde{F}=\left(\rho I+\bar{G}_{2}\right)^{-1}\left[\left(\rho I-\bar{G}_{1}\right)\left(\rho I+\bar{G}_{1}\right)^{-1} F+F\right] .
\end{aligned}
$$

Now define the matrix:

$$
\tilde{T}=\left(\rho I+\bar{G}_{2}\right) T\left(\rho I+\bar{G}_{2}\right)^{-1}
$$

which is similar to $T$ and thus has the same eigenvalues as $T$. Then it is evident that:

$$
\begin{gathered}
\rho(T)=\rho(\tilde{T})=\|\tilde{T}\|_{2} \leq \\
\left\|\left(\rho I-\bar{G}_{1}\right)\left(\rho I+\bar{G}_{1}\right)^{-1}\right\|_{2} \times\left\|\left(\rho I-\bar{G}_{2}\right)\left(\rho I+\bar{G}_{2}\right)^{-1}\right\|_{2} \\
=\max _{i}\left|\frac{\rho-\mu_{i}}{\rho+\mu_{i}}\right| \cdot \max _{i}\left|\frac{\rho-\eta_{i}}{\rho+\eta_{i}}\right| .
\end{gathered}
$$

Here $\mu_{i}, \eta_{i}(i=1,2, \cdots, J)$ are respectively the eigenvalues of $\bar{G}_{1}$ and $\bar{G}_{2}$.According to the lemmas, $\bar{G}_{1}$ and $\bar{G}_{2}$ are positive definite matrices, therefore $\mu_{i}>0, \eta_{i}>0(i=1,2, \cdots, J)$ and we have $\rho(T)<1$. Hence the convergence of the alternating iterative scheme is therefore proved.

\section{NUMERICAL EXPERIMENT}

The numerical examples of the sixth order AGI scheme are based on the following model problem: 


$$
\left\{\begin{array}{l}
-\frac{d^{2} u}{d x^{2}}+x u=\sin (\pi x)\left(x+\pi^{2}\right) \\
u(0)=u(2)
\end{array}\right.
$$

Where the exact solution is $u=\sin (\pi x)$ and the period is 2.

The error bounds of the sixth order AGI scheme are examined first. The $L^{2}$ errors are defined to be $e_{h}=\|u-u(x)\|_{L^{2}}$, and are computed based on the following four different $\rho$, and four different $h$ :

$$
\rho=2,3,4,5 ; h=\frac{2}{12}, \frac{2}{30}, \frac{2}{60}, \frac{2}{120} .
$$

The results are listed in Table 1 at the end of paper. It is not hard to see that the $L^{2}$ errors of the six-order scheme are order 6 in space, which confirms the earlier results in Section 2.

Next, we compare the accuracy of the sixth order AGI scheme to the fourth order AGI scheme. The computation is based on the same $\rho$, and $h$ is taken to be $\pi / 12$.The absolute errors (ae) and the percentage errors (pe) for these two schemes are listed in Table 2 and plotted at the end of paper. Evidently, the results show that the sixth order AGI scheme is more accurate than the fourth order AGI scheme.

TABLE I. CONVERGENCE RATES FOR THE SIXTH ORDER SCHEME

\begin{tabular}{|c|l|l|l|l|}
\hline \multirow{2}{*}{$J$} & \multicolumn{2}{|c|}{$\rho=1$} & \multicolumn{2}{c|}{$\rho=3$} \\
\cline { 2 - 5 } & $e_{h} * 10^{5}$ & $e_{h} / h^{6}$ & $e_{h} * 10^{7}$ & $e_{h} / h^{6}$ \\
\hline 12 & 3.012 & 0.001 & 301 & 0.001 \\
\hline 30 & 0.020 & 0.002 & 1.98 & 0.002 \\
\hline 60 & 1.579 & 11.98 & 0.044 & 0.003 \\
\hline 120 & 146 & 1463 & 0.017 & 8.21 \\
\hline \multirow{2}{*}{$J$} & \multicolumn{2}{|c|}{$\rho=5$} & \multicolumn{2}{c|}{$\rho=7$} \\
\cline { 2 - 5 } & $e_{h} * 10^{7}$ & $e_{h} / h^{6}$ & $e_{h} * 10^{7}$ & $e_{h} / h^{6}$ \\
\hline 12 & 301 & 0.001 & 301 & 0.001 \\
\hline 30 & 1.98 & 0.002 & 1.98 & 0.002 \\
\hline 60 & 0.044 & 0.003 & 0.044 & 0.003 \\
\hline 120 & 0.001 & 0.005 & 0.001 & 0.005 \\
\hline
\end{tabular}

\begin{tabular}{|c|c|c|}
\hline & \multicolumn{2}{|c|}{$\begin{array}{l}J=24, \quad \rho=2 \\
\Delta x=\pi / 12\end{array}$} \\
\hline & $x=0.26$ & $x=1.05$ \\
\hline \multicolumn{3}{|l|}{ 6th-order AGI } \\
\hline ae & $.133 \times 10^{-6}$ & $.244 \times 10^{-6}$ \\
\hline pe & $.517 \times 10^{-6}$ & $.282 \times 10^{-6}$ \\
\hline \multicolumn{3}{|l|}{ 4th-order AGI } \\
\hline ae & $.122 \times 10^{-4}$ & $.222 \times 10^{-4}$ \\
\hline pe & $.471 \times 10^{-4}$ & $.256 \times 10^{-4}$ \\
\hline \multirow[t]{4}{*}{ Exact solution } & 250 & 060 \\
\hline & \multirow{2}{*}{\multicolumn{2}{|c|}{$\begin{array}{l}J=24, \\
\Delta x=\pi / 12\end{array}$}} \\
\hline & & \\
\hline & $x=2.10$ & $x=3.40$ \\
\hline \multicolumn{3}{|l|}{ 6th-order AGI } \\
\hline ae & $.187 \times 10^{-6}$ & $.174 \times 10^{-7}$ \\
\hline pe & $.216 \times 10^{-6}$ & $.673 \times 10^{-7}$ \\
\hline \multicolumn{3}{|l|}{ 4th-order AGI } \\
\hline ae & $.170 \times 10^{-4}$ & $.159 \times 10^{-5}$ \\
\hline pe & $.196 \times 10^{-4}$ & $.614 \times 10^{-4}$ \\
\hline \multirow{4}{*}{ Exact solution } & .866 & -.259 \\
\hline & \multirow{2}{*}{\multicolumn{2}{|c|}{$\begin{array}{l}J=24, \quad \rho=2 \\
\Delta x=\pi / 12\end{array}$}} \\
\hline & & \\
\hline & $x=4.20$ & $x=6.02$ \\
\hline \multicolumn{3}{|l|}{ 6th-order AGI } \\
\hline ae & $.890 \times 10^{-7}$ & $.190 \times 10^{-7}$ \\
\hline pe & $.103 \times 10^{-6}$ & $.732 \times 10^{-7}$ \\
\hline \multicolumn{3}{|l|}{ 44th-order AGI } \\
\hline ae & $.811 \times 10^{-5}$ & \\
\hline pe & $.936 \times 10^{-5}$ & $.664 \times 10^{-5}$ \\
\hline Exact solution & $\begin{array}{l}-.866 \\
\end{array}$ & -.259 \\
\hline
\end{tabular}

TABLE II. ABSOLUTE AND PERCENTAGE ERRORS

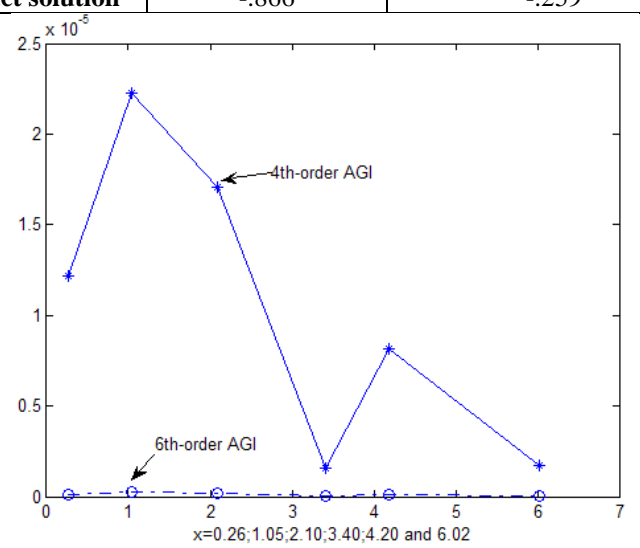

FIGURE I. ABSOLUTE ERRORS $J=24, \rho=2$ 


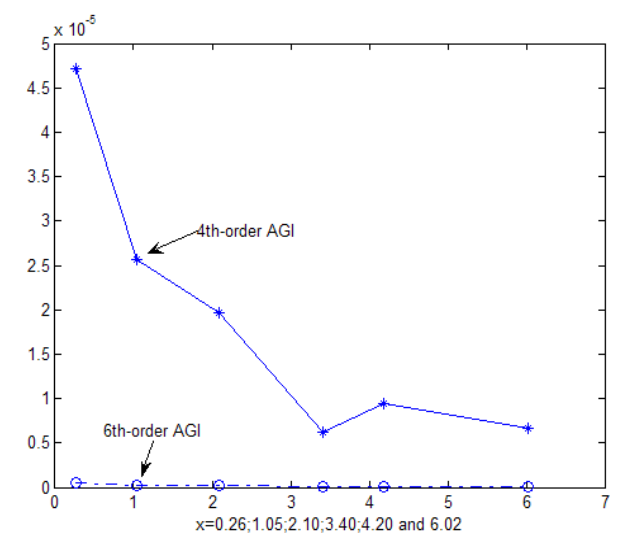

FIGURE II. PERCENTAGE ERRORS $J=24, \rho=2$

\section{CONCLUSIONS}

In this paper, a sixth order alternating group iterative algorithm is derived for two point boundary value problem. The scheme has truncation error of sixth order in space which is higher than similar fourth order AGI scheme. The scheme is proved to be stable under reasonable condition. Numerical example is also presented.

\section{ACKNOWLEDGEMENT}

This research was financially supported by the National Science Foundation of China (11501326).

\section{REFERENCES}

[1] D.J. Evans and A.R.B. Abdullah, Group explicit methods for parabolic equations. International Journal of ComputerMathematics, 14(1983), pp.73-105.

[2] D.J. Evans. Alternating Group Explicit (AGE) Iterative Methods. International Journal of Computer Mathematics,19(1986), pp.309-326.

[3] B.Zhang, Alternating segment explicit-implicit method for diffusion equation,Journal of Numerical Methods and Computational Applications, 12(1991),pp.245-251.

[4] D.J. Evans and A.R.B. Abdullah. a New explicit method for the Diffusion-Convection equation. Comput.Math.Appl.,11(1985), pp.145154.

[5] D.J. Evans. Group Explicit Iterative Methods for Solving Large Linear Systems. International Journal of Computer Mathematics., 17(1985), pp.81-108.

[6] S.Zhu and Jennifer Zhao,The Alternating Segment Explicit-Implicit Scheme for the Dispersive Equation,Letters in Applied Mathematics, 14(6),pp.657-662,2001.

[7] B.Zhang and W.Li, On alternating segment Crank-Nicolson scheme,Parallel Computing, 20(1994),pp.897-902.

[8] C.N.Dawson,Q.Du and T.F.Dupont,A finite difference domain decomposion algorithm for numerical solution of the Heat equation,Mathematical Computation, 57(1991), pp.63-71.

[9] Y.M.Shi,A high-order alternating group iterative algorithm,2011 Fourth International Joint Conference on Computational Sciences and Optimization,pp.12- 